\title{
Sodium Glucose Cotransporter-2 (SGLT-2) Inhibitor Related Diabetic Ketoacidosis
}

\author{
Atay Can Kula, MD \\ Emre Hoca, MD \\ Suleyman Ahbab, MD, Assoc. Prof. \\ H. Esra Ataoglu, MD, Assoc. Prof.
}

University of Health Sciences, Istanbul Haseki Health Training and Research

Hospital, Internal Medicine Clinic, Istanbul, Turkey

Doi: 10.19044/esj.2019.v15n15p268 URL:http://dx.doi.org/10.19044/esj.2019.v15n15p268

\begin{abstract}
Diabetic ketoacidosis is a rare but serious and life-threatening acute complication of diabetes mellitus. It is characterized by ketoacidosis with other findings and should be treated immediately. In this case, a 33-year-old diabetic patient who admitted to the emergency department with diabetic ketoacidosis is presented. The patient was prescribed empagliflozin (sodium glucose cotransporter inhibitor-2) a month ago. The SGLT-2 inhibitors have been approved for use in the treatment of type $2 \mathrm{DM}$ and are still not used in the treatment of type $1 \mathrm{DM}$. There are such reports of unusual side effects related SGLT-2 inhibitors, in the literature and among them, ketoacidosis is a rare and important side effect.
\end{abstract}

Keywords: SGLT-2 Inhibitor, Diabetes Mellitus Treatment, Diabetic Ketocidosis

\section{Introduction}

Diabetic ketoacidosis (DKA) is a serious and life-threatening acute complication of diabetes mellitus. It is characterized by ketoacidosis and hyperglycemia. Sodium-glucose cotransporter 2 (SGLT-2) inhibitors decrease plasma glucose levels by blocking the reabsorption of glucose from the proximal tubules (Zinman B et al., 2015). SGLT-2 inhibitors are indicated as an add-on therapy when a single agent is inadequate to achieve glycemic goals in type $2 \mathrm{DM}$. Several studies have shown beneficial glycemic effects of SGLT-2 inhibitors in selected type 1 DM patients. (Plosker GL.2014, Sha S et al. 2011). However, regulatory agencies (as FDA) have not approved this class of drugs for type 1 diabetic patients, yet. Some case reports suggest that SGLT2 inhibitors may be attributed to an increased risk of diabetic ketoacidosis 
(Ogawa W et al., 2016). We aimed to emphasize diabetic ketoacidosis as a rare potential complication of Empagliflozin treatment. In this case, we present a patient who developed diabetic ketoacidosis after using SGLT-2 inhibitor.

\section{Case}

A 33-years-old female patient admitted to our hospital with nausea, vomiting and mild abdominal pain for the past 2 days. She was diagnosed in an another health center with insulin dependent diabetes mellitus (DM) 4 years ago and had mental retardation. Patient's oral intake was inordinate due to mental retardation. The patient had no fever, alcohol intake history, smoking or surgical intervention history. As her symptoms became worse, she applied to the emergency clinic of our hospital. She was treated with Insulin Glargine and Insulin Glulisine for 4 years. Empagliflozin treatment (10 mg per day) was started one month ago. Vital signs at the time of admission were as follows; temperature $36.3{ }^{\circ} \mathrm{C}$, pulse rate 120 beats/min, respiratory rate 28 breaths $/ \mathrm{min}$ and blood pressure $153 / 89 \mathrm{~mm} \mathrm{Hg}$. The patient was dehydrated with sunken eyes, dry oral mucosa and poor skin turgor. The patient had thoraco-abdominal pattern of breathing. Auscultation of the lungs revealed no significant findings. Arterial blood gases showed severe metabolic acidosis with an elevated anion gap (pH 7.04, CO2 $16.8 \mathrm{mmHg}, \mathrm{HCO} 36.2 \mathrm{mEq} / \mathrm{L}$, anion gap $28.1 \mathrm{mEq} / \mathrm{L}$ ). Hyperglicemia (419 mg/dL), ketonuria and glucosuria were also detected. Serum lactate level was normal $(1.55 \mathrm{mg} / \mathrm{dL})$. Coagulation parameters (INR, prothrombin time), liver enzymes, renal functions, serum protein, albumin and other laboratory findings were normal. Chest radiogram and electrocardiogram showed no significant findings. She was hospitalised in the intensive care unit with diabetic ketoacidosis. The patient was rehydrated with intravenous fluid solutions and electrolyte replacement was given. The patient's Empagliflozin treatment was discontinued. Serial arterial blood gas analyses showed an apperent improvement on ketoacidosis after the treatment. The follow up is being continued in our clinic.

\section{Discussion}

DKA is characteristically associated with type 1 diabetes. It also occurs in type 2 diabetes under conditions of extreme stress such as serious infection, trauma, cardiovascular or other emergencies and less often, as a presenting manifestation of type 2 diabetes. The most widely used diagnostic criteria for diabetic ketoacidosis include blood glucose $>250 \mathrm{mg} / \mathrm{dL}$, arterial $\mathrm{pH}<7.3$, serum bicarbonate $<15 \mathrm{mEq} / \mathrm{L}$ and a moderate degree of ketonemia and/or ketonuria (Kitabchi et al, 2001). DKA is more common in younger $(<65$ years) patients (Benoit et al, 2018). Empagliflozin is a selective SGLT-2 inhibitor which can be used either as a single treatment or in combination with 
other antidiabetics. It is approved by the FDA (U.S. Food and Drug Administration) in 2014 for type 2 diabetic patients.. This drug increases the amount of glucose excreted in urine and beneficial for lowering the blood glucose level. It provides a reduction in required insulin doses for type 2 diabetic patients. SGLT-2 inhibitors that are given with insulin control hyperglycemia in type 2 diabetes mellitus without increasing the risk of hypoglycemia.

It was found that SGLT-2 inhibitors cause an increase in urinary tract infection, bladder cancer, acute renal failure, bone fracture and diabetic ketoacidosis frequencies (Musso et al, 2012). Also, in some of the diabetic patients using SGLT-2 inhibitor, an increase in the incidence of euglycemic diabetic ketoacidosis was observed (Taylor et al, 2018). Since 2015, several cases of DKA associated with SGLT-2 inhibitors were reported both in type 1 DM and type 2 DM (Taylor et al, 2018-Rosenstock J. et al, 2015). Type 1 DM patients who treated with SGLT-2 inhibitors could achieve glucose regulation but ketoacidosis frequency increases in this patients. SGLT2 inhibitors enhance risk of ketogenesis and lead to DKA, especially in susceptible diabetic patients and in the presence of precipitating factors (Fattah, H.et al, 2018). Insulin-independent glucose clearance, hyperglycaemia, and volum deficiency are implicated as mechanisms (Peters et al, 2015). As seen in our case, patients using SGLT-2 inhibitors who have nausea, vomiting, and reduced oral intake should be carefully examined for diabetic ketoacidosis. The use of SGLT-2 inhibitors in patients with type 1 diabetes is considered to be non-indicative and is not suitable for use in these patients. Experiences with Empagliflozin associated DKA are still limited. There are still no sufficient data on this subject and further clinical studies are needed (Ahmed-Sarwar et al, 2017).

\section{References:}

1. Zinman B, Wanner C, Lachin JM, et al. (2015) Empagliflozin, cardiovascular outcomes, and mortality in type 2 diabetes. $\mathrm{N}$ Engl J Med; 373:211728.

2. Plosker GL.(2014) Canagliflozin: a review of its use in patients with type 2 diabetes mellitus. Drugs. ;74:807-824.

3. Sha S, Devineni D, Ghosh A, et al. (2011)Canagliflozin, a novel inhibitor of sodium glucose co-transporter 2, dose dependently reduces calculated renal threshold for glucose excretion and increases urinary glucose excretion in healthy subjects. Diabetes Obes Metab. ;13:669672.

4. Ogawa W, Sakaguchi K. (2016) Euglycemic diabetic ketoacidosis induced by SGLT2 inhibitors: possible mechanism and contributing factors. J Diabetes Investig; 7:1358. 
5. Kitabchi AE, Umpierrez GE, Murphy MB, et al. (2001) Management of hyperglycemic crises in patients with diabetes. Diabetes Care; 24: 131-153.

6. Benoit SR, Zhang Y, Geiss LS, et al. (2018) Trends in Diabetic Ketoacidosis Hospitalizations and In-Hospital Mortality - United States, 2000-2014. MMWR Morb Mortal Wkly Rep; 67:362.

7. Musso G, Gambino R, Cassader M, et al. (2012). A novel approach to control hyperglycemia in type 2 diabetes: SGLT inhibitors. Systematic reviews metaanalysis of randomized trials. Ann Med. 44(4):375-93. doi: 10.3109/07853890.2011.560181

8. Taylor SI, Blau JE, Rother KI. (2018) SGLT2 Inhibitors May Predispose to Ketoacidosis. The Journal of Clinical Endocrinology \& Metabolism. doi: 101210/jc.2015-1884.

9. Jaberi A, Seth B, Steenkamp, Alexanian S, Borkan SC.(2016) Sodiumglucose cotransporter 2 inhibitors and euglycemic diabet- ic ketoacidosis: metabolic acidosis with a twist. Clin Diabetes. ;34:214216.

10. Rosenstock J, Ferrannini E. (2015) Euglycemic diabetic ketoacidosis: a predictable, detectable, and preventable safety concern with SGLT2 inhibitors. Diabetes Care.;38:1638-1642.

11. Fattah, H., \& Vallon, V. (2018). The Potential Role of SGLT2 Inhibitors in the Treatment of Type 1 Diabetes Mellitus. Drugs, 78(7), 717-726. doi:10.1007/s40265-018-0901-y

12. Peters AL, Buschur EO, Buse JB, et al. (2015) Euglycemic Diabetic Ketoacidosis: A Potential Complication of Treatment With SodiumGlucose Cotransporter 2 Inhibition. Diabetes Care. 38(9):1687-93.

13. Ahmed-Sarwar N, Nagel AK, Leistman S, Heacock K. (2017) SGLT2 Inhibitors: Is There a Role in Type 1 Diabetes Mellitus Management? Ann Pharmacother 51(9):791-796. doi: 10.1177/1060028017710481 